\title{
Speciation of organotin compounds in environmental samples with semi-permanent coated capillaries by capillary electrophoresis coupled with inductively coupled plasma mass spectrometry $\dagger$
}

\author{
Jing Sun, Bin He, ${ }^{*}$ Yongguang Yin, Lu Li and Guibin Jiang \\ Received 15th September 2010, Accepted 22nd September 2010 \\ DOI: $10.1039 / \mathrm{c0ay00558d}$
}

A novel method using capillary electrophoresis (CE) coupled with inductively coupled plasma mass spectrometry (ICP-MS) for the separation of four organotin compounds has been developed. Semipermanent coating of dioctadecyl dimethyl ammonium chloride (DDAC) was applied and investigated to enhance separation efficiency of trimethyltin (TMT) chloride, tributyltin (TBT) chloride, dibutyltin (DBT) dichloride and monobutyltin (MBT) trichloride. The electrolyte composition and ionic strength were optimized. Running buffer containing $0.608 \mathrm{~mol} \mathrm{~L}^{-1} \mathrm{HAc}$ and $7 \mathrm{mmol} \mathrm{L}{ }^{-1} \mathrm{NaAc}$ was selected for the separation. The detection limits of the four organotin compounds ranged from 0.037 to $0.112 \mathrm{mg} \mathrm{L}^{-1}$ (as Sn) and the values of the relative standard deviation (RSD) were below $3.1 \%$ for migration time, less than $7.8 \%$ for peak height and not more than $8.4 \%$ for peak area. The developed method was successfully applied for speciation analysis of four organotin compounds in one certified reference material (CRM 477, mussel tissue), and environmental samples including water samples, grape wine and biological samples (Mya arenaria Linnaeus). The determined values (as organotin compounds) of CRM 477 (1.81 \pm 0.13 (TBT), $1.36 \pm 0.16$ (DBT), $1.17 \pm 0.18$ (MBT)) were in good agreement with the certified values $(2.20 \pm 0.19$ (TBT), $1.54 \pm 0.12$ (DBT), $1.50 \pm 0.28$ (MBT)).

$0.325 \mathrm{mg} \mathrm{L}^{-1}$ MBT and $1.02 \mu \mathrm{g} \mathrm{g}^{-1}$ (as Sn) TBT were found in grape wine and Mya arenaria Linnaeus samples.

\section{Introduction}

Organotin compounds are important organometallic pollutants, which are mainly used as antifouling paints, PVC stabilizers, pesticides and additives in many industrial, chemical and agricultural applications. ${ }^{1}$ The growing consumption of tin compounds in recent years result in a great amount of organotin compounds widely detected in environmental matrixes such as seafood and wine. ${ }^{2}$ In addition, high toxicity of the organotin compounds raised increasing concerns over their existence in the environment. The toxicity of organotin compounds is strongly dependent on the type and the number of alkyl groups linked to the tin atom. ${ }^{3,4}$ Although the inorganic tin is thought to be harmless, TBT (tributyltin), TPhT (triphenyltin), and some other organotin compounds have already been proved to be harmful to non-target aquatic organisms even at a low ng $\mathrm{L}^{-1}$ level. ${ }^{5}$ Thus, separation and determination of organotin compounds in various matrixes are of great importance in studies on environmental pollution and ecotoxicological concerns.

The technique for the separation and quantification of organotin compounds should be selective and sensitive. There are already many methods which have been used to separate and monitor organotin compounds in environmental samples. The most common technique to separate organotin derivatives is gas

State Key Laboratory of Environmental Chemistry and Ecotoxicology, Research Center for Eco-Environmental Sciences, Chinese Academy of Sciences, P.O. Box 2871, Beijing, 100085, China. E-mail: bhe@rcees.ac. cn; Fax: +86-10-62849179; Tel: +86-10-62849334

$\dagger$ Electronic supplementary information (ESI) available: Table S1-S4 and Fig. S1-S4. See DOI: 10.1039/c0ay00558d chromatography (GC) coupled with flame photometric detection (FPD), ${ }^{6,7}$ atomic absorption spectrometry (AAS), ${ }^{8}$ pulsed flame photometric detection (PFPD), ${ }^{9}$ and $\mathrm{MS} .{ }^{10}$ GC related techniques could offer excellent resolution and low detection limit. Whereas the major drawback relates to the poor volatility of the organotin compounds and a pre-column derivatization step is needed for the GC analysis, which is tedious and time consuming. ${ }^{11}$ Meanwhile, a high performance liquid chromatography (HPLC) technique has also been used to separate these organotin compounds. HPLC coupled with inductively coupled plasma mass spectrometry (ICP-MS) and mass spectrometry have been conducted for environmental organotins determination. ${ }^{12}$ Although the ICP-MS and ICP-OES detectors are sensitive, these equipments are critical for LC mobile phases so that LC separation parameters such as the concentration of the organic mobile phase and the proportion of the salt in the mobile phase should be restricted.

Over the last two decades, there are increasing concerns using capillary electrophoresis (CE) for the separation of organometallic compounds in various complex matrixes. The $\mathrm{CE}$ technique has the advantage of high separation efficiency, rapid analysis, and little volume of sample consumption. CE coupled to inductively coupled plasma-mass spectrometry is an attractive method for separating and quantifying organometallic compounds. ICP-MS can offer not only elemental information of the compounds, but also offers excellent sensitivity, a wide linear dynamic range, high-speed analysis and the ability to perform isotopic analysis, ${ }^{11}$ compared with other previous publications with UV and fluorescence detectors. ${ }^{13-16}$ All these advantages bring CE-ICP-MS into the central focus of element speciation, 
where typically the separation of species is followed by elementselective detection.

The adsorption of organotin compounds on the inner wall of capillaries is a challenge for the CE method, which might result in poor migration time reproducibility, low sample recovery and peak tailing. No obvious improvements were reported even approaches such as high ionic strength and extreme $\mathrm{pH}$ were applied in the separation optimization procedures. ${ }^{17}$ Capillary inner wall coating technique was recognized and widely used as an effective way to inhibit analyte adsorption on capillaries and thus offer lower method determination limits. The double-chained cationic surfactant dioctadecyl dimethyl ammonium chloride (DDAC) and didodecyldimethylammonium bromide (DDAB) have been found to form more stable coatings onto the walls of CE capillaries. These surfactants can be reversibly adsorbed onto the negatively charged wall, reverse the surface charge and affect the capillary electroosmotic flow (EOF) when their concentrations are above the critical micelle concentration. Moreover, coating of semipermanent DDAC and DDAB are robust and simple by flushing these aqueous solutions through the capillary for only several minutes. No further adsorption materials are needed in the running buffer. The stability of the semi-permanent coatings of DDAC and DDAB has also been confirmed by the previous articles which were excellent for $\mathrm{CE}$ separations over a 12-day period without ever regenerating or refreshing the capillary. ${ }^{18}$ There are many applications reported using DDAB and DDAC as capillary inner wall modifiers. ${ }^{19,20}$ However, these techniques have not been applied for the CE separation of organotin compounds.

In this paper, we describe a method using DDAC coating-CE coupled with ICP-MS to separate four organotin compounds (TMT, TBT, DBT, MBT). Acetic acid and sodium acetate were introduced into the electrolyte running buffer with certain ionic strength. The effect of concentration and composition of the background electrolyte on separation and detector response were examined and the applications of the method to several environmental samples were also conducted.

\section{Experimental}

\subsection{Materials, chemicals, and samples}

Trimethyltin (TMT) chloride, tributyltin (TBT) chloride, dibutyltin (DBT) dichloride were purchased from Dr Ehrenstorfer GmbH (Augsburg, Germany), monobutyltin (MBT) trichloride was obtained from Acros (Geel, Belgium). GR grade acetic acid was from Beijing Chemical Co. (Beijing, China) and sodium acetate $(\mathrm{NaAc})$ was from Sinopharm Chemical Reagent Co., Ltd (Shanghai, China). Distilled deionized water (DDW) (18 M $\Omega . c m$ ) was obtained from Milli-Q Advantage A10 system (Millipore, Bedford, MA). Dioctadecyl dimethyl ammonium chloride and nitric acid (69\% purity) were obtained from SigmarAldrich (Steinheim, Germany). The standard mussel tissue reference material (CRM 477) was obtained from the Community Bureau of Reference, Commission of the European Communities. All other compounds used in the experiment were at least of analytical-reagent grade.

\subsection{Preparation of buffer and standard solutions}

The stock solutions of organotin compounds were prepared by dissolving TMT, TBT, DBT and MBT in HPLC-grade methanol to obtain a solution with the concentration of $1 \mathrm{mg} \mathrm{mL}^{-1}$ (as $\mathrm{Sn}$ ). The working standard solutions were freshly prepared by diluting the stock solutions with DDW, and the $\mathrm{pH}$ of the solutions was adjusted to 2 by using concentrated $\mathrm{HCl}$ to ensure the stability of the solutions. Both stock solutions and working standard solutions were store at $4{ }^{\circ} \mathrm{C}$ in the dark.

The electrolyte running buffer containing HAc and $\mathrm{NaAc}$ in series were prepared by gradually dilution with DDW. The sheath flow was composed of $1 \%(\mathrm{v} / \mathrm{v})$ nitric acid. DDAC was dissolved in DDW to a concentration of $15 \mu \mathrm{mol} \mathrm{L}^{-1}$. The electrolyte running buffer, sheath flow liquid, and DDAC solutions were filtrated through $0.45 \mu \mathrm{m}$ membrane before use.

\subsection{Semi-permanent coating and capillary conditioning}

Every new capillary was conditioned by flushing with $1 \mathrm{~mol} \mathrm{~L}{ }^{-1}$ $\mathrm{NaOH}$ for $60 \mathrm{~min}, 0.1 \mathrm{~mol} \mathrm{~L}^{-1} \mathrm{NaOH}$ for $60 \mathrm{~min}$, DDW for $10 \mathrm{~min}$, and then, by electrolyte running buffer for $60 \mathrm{~min}$. Then semi-permanent coating was generated by flushing a $0.1 \mathrm{mM}$ DDAC water solution through the capillary for $20 \mathrm{~min}$.

After each run of separation, the capillary was flushed with $0.1 \mathrm{~mol} \mathrm{~L}^{-1} \mathrm{NaOH}$ for $2 \mathrm{~min}, 15 \mu \mathrm{M}$ DDAC solution was used for $1 \mathrm{~min}$ to guarantee the stability of the DDAC coating, and finally with the running buffer solution for $3 \mathrm{~min}$.

\subsection{CE operating conditions}

All the CE separations of these four organotin compounds were operated on a $\mathrm{HP}^{3 \mathrm{D}} \mathrm{CE}$ system (Agilent, Germany) at a voltage of $20 \mathrm{kV}$. The samples were introduced into the capillary using a hydrodynamic method under a gas presser of 20 mbar for $5 \mathrm{~s}$. The fused-silica capillaries used in the experiment were $60 \mathrm{~cm}$ long with an inner diameter of $75 \mu \mathrm{m}$, which were obtained from Yongnian Optical Fiber Company (Hebei, China).

\subsection{ICP-MS system}

An Agilent 7500ce ICP-MS instrument (Agilent, USA) was coupled with the $\mathrm{HP}^{3 \mathrm{D}} \mathrm{CE}$ system and used for the quantification. A MicroMist nebulizer (AR35-1-FM005E, GE, Australia) was used to fit on the standard spray chamber. A standard torch was also used with a plasma gas flow rate of $15 \mathrm{~L} \mathrm{~min}^{-1}$, carrier gas flow rate of $0.7 \mathrm{~L} \mathrm{~min}^{-1}$ and the makeup gas flow rate of $0.4 \mathrm{~L} \mathrm{~min}^{-1}$. The plasma RF power was $1500 \mathrm{~W}$. Signals from the four isotopes $\left({ }^{117} \mathrm{Sn},{ }^{118} \mathrm{Sn},{ }^{119} \mathrm{Sn},{ }^{120} \mathrm{Sn}\right)$ of tin were collected in FullQuant mode. The integration time was $0.3 \mathrm{~s}$ per mass. Complete information of the ICP-MS system is shown in Table S1. $\dagger$

\subsection{Interface of CE-ICP-MS system}

Interfaces of CE coupled to ICP-MS were considered as a key part in the hyphenation technique. ${ }^{21,22}$ The interface used in the present study has been published elsewhere. ${ }^{23-25}$ In brief, a homemade interface of a cross design, introducing a Pt electrode (0.8 mm diameter), CE capillary and a sheath flow, was chosen and used throughout the sample analysis. The CE capillary was threaded through the cross fitting and was inserted into the 
MicroMist nebulizer. The sheath flow was used to wet the outlet of capillaries and satisfy the demands of the closing of the electrical circuit from CE. The Pt electrode was grounded, and the sheath flow was transported into the nebulizer in the self-aspiration mode with a liquid take-up of approximately $50 \mu \mathrm{L} \mathrm{min}{ }^{-1}$. To reduce the broadening of the sample peaks, the CE capillary was inserted to the end of the MicroMist nebulizer through a 1/16-inch PEEK tube. The outlet end of the CE capillary was positioned $5 \mathrm{~cm}$ from the end tip of the nebulizer.

\subsection{Extraction procedure and sample preparation}

The river water used for the experiment was obtained from Liuyang River (Hunan, China) and the tap water was collected stochastically from the laboratory. The dry red wine sample used in the experiment was obtained from a local market. All these liquid samples were filtrated through $0.45 \mu \mathrm{m}$ membranes twice and then used for the analysis.

The microwave extraction procedure for organotin compounds from mussel tissue samples was used as published elsewhere ${ }^{26}$ with little modification. Briefly, $0.5 \mathrm{~g}$ of lyophilized Mya arenaria Linnaeus sample and CRM 477 (mussel tissue) were weighted and supplemented in the vessels with $10 \mathrm{~mL}$ of methanol containing $0.5 \mathrm{~mol} \mathrm{~L}^{-1}$ acetic acid. Then the vessels were submitted to the microwave-assisted extraction system (Mars5 HP500, CEM Corporation, Matthews) at $60 \mathrm{~W}$ for $3 \mathrm{~min}$. After cooling, the samples were transferred into glass centrifuge tubes and centrifuged at $3000 \mathrm{rpm} / \mathrm{min}$ for $5 \mathrm{~min}$. The supernatant liquid were collected and then evaporated almost to dryness under a gentle stream of nitrogen. Subsequently, the residue was diluted to $4.0 \mathrm{~mL}$ with electrolyte buffer (HAc : $\mathrm{NaAc}=608 \mathrm{mM}: 7 \mathrm{mM}$ ). The emulsions were broken up by subjecting the tubes to be centrifuged at $3000 \mathrm{rpm} / \mathrm{min}$ for $5 \mathrm{~min}$ again. The supernatant liquid was finally gathered, filtered through a $0.22 \mu \mathrm{m}$ membrane and analyzed by CE-ICP-MS.

\section{Results and discussion}

\subsection{Optimization of $\mathrm{CE}$ conditions}

For the optimization, several operation parameters such as concentration of electrolyte and composition of electrophoretic buffer were tested in detail. Initial experiments were performed by using acidic aqueous solutions. The addition of HAc has been proved to be necessary to increase the detector response of organotin compounds. Several other electrophoretic buffers have also been examined, including tartaric acid-methanol buffer, $\mathrm{Na}_{2} \mathrm{HPO}_{4}$-boric acid buffer and boric acid-Tris buffer (Fig. S1). $\dagger$ However, it was found that only acetic buffer could offer a good electropherogram, along with the best resolution. Thus, acetic buffer was selected for the following experiments.

3.1.1 Semi-permanent coating. Primary experiments showed that the organotin compounds were strongly adsorbed to the inner wall of the capillaries and resulted in bad peak shapes and the phenomenon could usually be attributed to the structures of the organotin compounds. ${ }^{27} \mathrm{MBT}$ and DBT own only one or two n-butyl groups independently which attached to the tin atom and were +3 or +2 charged respectively. Both the steric hindrance and more positive charges made MBT and DBT more favorable to interact with the ionized silanol groups on the inner-wall of the capillary.

As a cationic surfactant, DDAC coating can form a bilayer or vesicle structure at the inner walls of capillaries, changing the ionized silanol groups on the capillary inner wall into a positively charged surface, which can prevent the organotin compounds from adsorption onto the ionized silanol groups on the inner wall of the uncoated capillary. Semi-permanent coating of DDAC is simple and stable. The stability of DDAC coatings owes to the formation of the bilayer or vesicle structure at the inner wall of capillaries, which were flatter and more homogeneous, resulting in not only better stability but also greater surface coverage to prevent the adsorption of the organotin compounds. The separation efficiency of coating and uncoating capillaries could be compared in Fig. 1. It was found that with semi-permanent coating of DDAC, the migration times of organotins have been affected obviously. The four organotin compounds (TMT, TBT, DBT, and MBT) could achieve baseline separation in $12 \mathrm{~min}$ and the peak areas were increased when coated capillaries were used. Also shown in Table 1, the migration times of the analytes with coated capillaries were stable with the relative standard deviation ranging from $2.1 \%$ to $3.1 \%$ (intra-day) and $2.4-3.4 \%$ (inter-day).

3.1.2 Effect of buffer concentration. For separation of these four organotin compounds, ionic strength of the running buffer was investigated. The results in Fig. 2 showed that the sensitivity of organotin compounds was improved significantly with the increase of buffer concentration from the range of $102.5 \mathrm{mM}$ to $820 \mathrm{mM}$. But when the concentration was above $820 \mathrm{mM}$, migration times of TBT and TMT were getting closer to each other that the baseline separation was not achieved. Thus the running buffer at the concentration of $615 \mathrm{mM}$ was chosen for the baseline separation as well as detection response improvement.

3.1.3 Effect of the electrolyte buffer composition. It has been reported that the buffer composition affects the separation

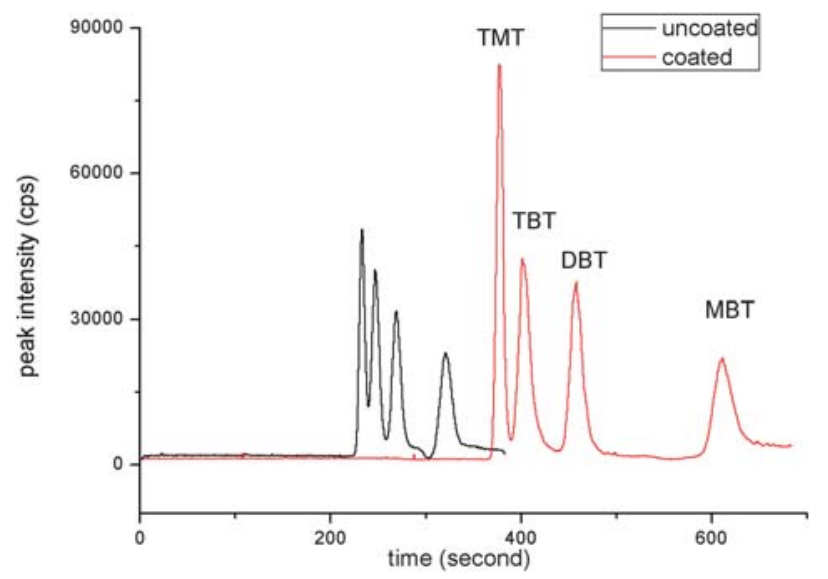

Fig. 1 Comparison of electropherogram of semi-permanent coated and uncoated capillaries. Electropherograms: (1) separation of organotin compounds with DDAC coating; (2) separation of organotin compounds without DDAC coating. Electrolyte running buffers: $0.608 \mathrm{~mol} \mathrm{~L}^{-1} \mathrm{HAc}$ and $7 \mathrm{mmol} \mathrm{L}^{-1} \mathrm{NaAc}$. Other conditions: $20 \mathrm{kV}, 25^{\circ} \mathrm{C}, 20 \mathrm{mbar} \times 5 \mathrm{~s}$ injection. Concentration of organotin compounds: $1 \mu \mathrm{g} \mathrm{mL}^{-1}$ as $\mathrm{Sn}$. 
Table 1 Analytical performance of four organotin compounds under optimized condition by CE-ICP-MS

\begin{tabular}{|c|c|c|c|c|c|c|c|}
\hline \multirow[b]{2}{*}{ Compound } & \multicolumn{4}{|l|}{ RSD (\%) } & \multirow[b]{2}{*}{ Linear range/mg L $\mathrm{L}^{-1}$} & \multirow[b]{2}{*}{$R^{2}$} & \multirow[b]{2}{*}{$\mathrm{LOD} / \mathrm{mg} \mathrm{L}^{-1}$} \\
\hline & Height $(n=5)$ & Area $(n=5)$ & Intra-day $(n=5)$ & Inter-day $(n=12)^{a}$ & & & \\
\hline TMT & 5.0 & 7.1 & 2.1 & 2.4 & $0.037-1.000$ & 0.9950 & 0.037 \\
\hline TBT & 2.8 & 6.3 & 2.3 & 2.7 & $0.062-1.000$ & 0.9907 & 0.062 \\
\hline DBT & 5.7 & 8.4 & 2.3 & 2.7 & $0.084-1.000$ & 0.9886 & 0.084 \\
\hline
\end{tabular}

greatly, since it could influence the electrophoretic mobility of the cations. The composition of the electrolyte buffer has been considered. It was found that in Fig. 3, with the decreasing ratio of HAc : NaAc from $84: 1$ to $14: 1$, the migration times of the four organotin compounds were getting closer. However, when the electrolyte buffer was HAc only, migration times of TBT and DBT are closer, which could not achieve baseline separation. So the proportion of the electrolyte running buffer was chosen as $84: 1$ and the concentrations of $\mathrm{NaAc}$ and HAc were $7 \mathrm{mM}$ and $608 \mathrm{mM}$, respectively.

3.1.4 Effect of injection time. Compared with electrokinetic sample injection, hydrodynamic injection is not critical to the species and samples and do not result in systematic errors for the analysis. So hydrodynamic injection was chosen as the sample injection mode. As sample injection time has a significant effect on the detection response, a series of injection times of $1 \mathrm{~s}, 3 \mathrm{~s}, 5 \mathrm{~s}$, $10 \mathrm{~s}$, and $15 \mathrm{~s}$ were investigated under the hydrodynamic injection pressure of $20 \mathrm{mbar}$ (Fig. S2). $\dagger$ When the injection time was increased from 1 to $15 \mathrm{~s}$, the peak areas of the organotin compounds were increased significantly. However, the widths of the peaks were continually broadened, and the baseline separation could not be achieved even when the injection time was up to $10 \mathrm{~s}$. Under the circumstances, the injection time of $5 \mathrm{~s}$ was chosen and thus the volume of sample injection was about $2.1 \mathrm{~nL}$.

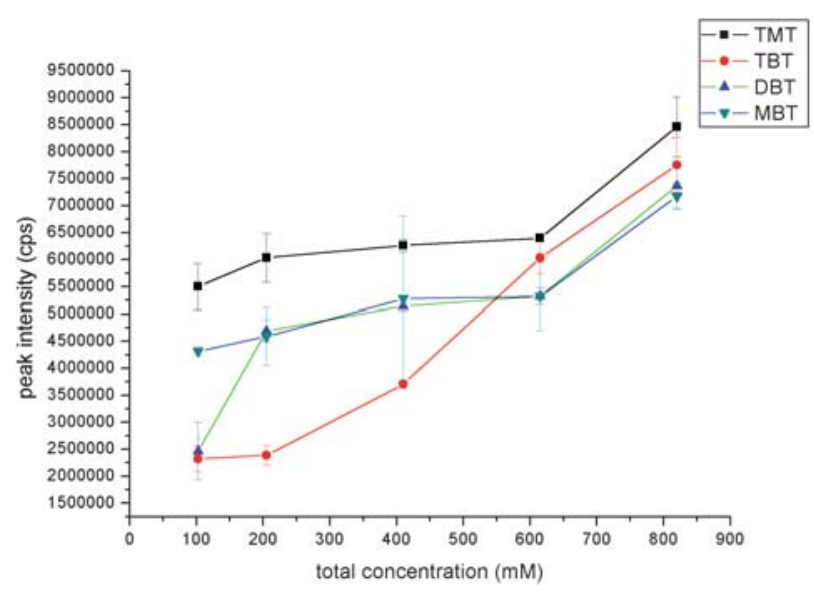

Fig. 2 Effect of buffer concentration in mobile phase on the organotin compounds peak intensity. The data was obtained by determining $1 \mathrm{mg} \mathrm{L}^{-1}$ mixed solution of TMT, TBT, DBT and MBT with CE-ICP-MS under optimized condition except buffer concentration.
3.1.5 Effect of $\mathrm{HNO}_{3}$ concentration in the make up solution. Self-aspiration was used as uptake mode of the sheath flow solution in this work. Self-aspiration of the sheath flow liquid can minimize or eliminate the laminar flow completely. ${ }^{22}$ Nitric acid was proved to be the best choice as the make up solution, which can avoid potential disadvantages such as plasma instability, poor precision, and degradation of ICP-MS performance.

The effects of $\mathrm{HNO}_{3}$ concentration on the intensity of ICP-MS signals of organotins were investigated in the range of $0.1 \%-3 \%$ (v/v). As shown in Fig. S3, $\dagger$ it was obvious that the peak areas increased when the concentration of $\mathrm{HNO}_{3}$ was raised from $0.1 \%$ to $1 \%$. Whereas the intensity decreased when the $\mathrm{HNO}_{3}$ concentration was further increased from $1 \%$ to $3 \%$. This phenomenon could be explained by the viscosity of sheath flow solution and nebulization efficiency of the nebulizer. When $\mathrm{HNO}_{3}$ concentration rose to $1 \%$, the increased peak intensity might be caused by the increasing viscosity of sheath flow, which made the $\mathrm{HNO}_{3}$ sheath flow slower, resulting in improvement of transfer efficiency. However, when the concentration of $\mathrm{HNO}_{3}$ increased from $1 \%$ to $3 \%$, the viscosity still increased but nebulization efficiency was depressed significantly. Thus, the corresponding peak intensity became lower. As a result, 1\% (v/v) $\mathrm{HNO}_{3}$ was chosen as the optimized sheath flow solution and also used to remove equipment memory effect.

\subsection{Separation of organotin compounds under the optimized condition}

With the optimized analysis parameters, TMT, TBT, DBT, and MBT were all baseline separated. A series of experiments were performed and linearity, limit of detection (LOD), precision and accuracy were validated to value the quantification performance of the method.

3.2.1 Linearity, concentration range and limit of detection. The linear regression equations were obtained by using five concentration levels $\left(0.05,0.1,0.2,0.5\right.$ and $1 \mathrm{mg} \mathrm{L}^{-1}$ as $\left.\mathrm{Sn}\right)$ of the mixture solutions of the four analytes. Each level was tested in triplicate under the optimized condition. The linearity and the concentration range resulting from the regressions are given in Table 1 . The results showed good linearity $\left(R^{2} \geq 0.9886\right)$ in the range of $0.05 \mathrm{mg} \mathrm{L}^{-1}-1.00 \mathrm{mg} \mathrm{L}^{-1}$ for the four organotin compounds.

Investigation of detection limits (LOD) of the method was based on the data used for linearity and precision. The LODs of method have been calculated based on 3 times of $\mathrm{S} / \mathrm{N}$ ratio. From 

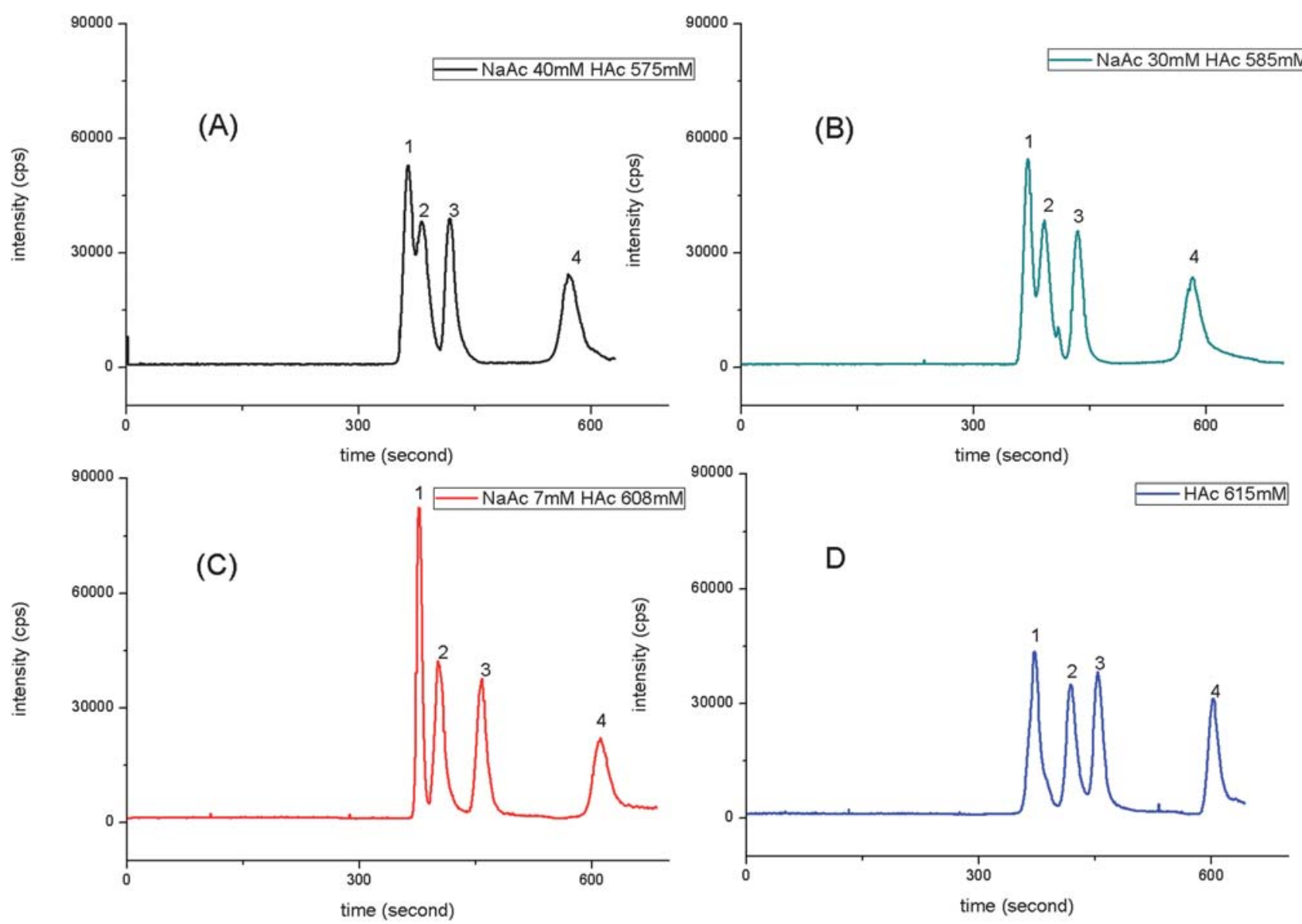

Fig. 3 Effect of electrolyte buffer composition in mobile phase on the organotin compounds. The data was obtained by determining $1 \mathrm{mg} \mathrm{L}^{-1}$ mixed solution of TMT, TBT, DBT and MBT with CE-ICP-MS under optimized condition except electrolyte buffer composition. Electrolyte buffer composition: (A) NaAc: $40 \mathrm{mM}$ and HAc: $575 \mathrm{mM}$; (B) NaAc: $30 \mathrm{mM}$ and HAc: $585 \mathrm{mM}$; (C) NaAc: $7 \mathrm{mM}$ and HAc: $608 \mathrm{mM}$; (D) HAc: $615 \mathrm{mM}$. Legend: 1, TMT; 2, TBT; 3, DBT; and 4, MBT.

Table 1, it was shown that the LODs of the four organotin compounds ranged from 0.037 to $0.112 \mathrm{mg} \mathrm{L}^{-1}$. The developed method has lower LODs than the published CE-hyphenated techniques (Table S2) $\dagger$ As shown in Fig. 1, DDAC coating inhibited the adsorption of organotin compounds on the inner wall of capillaries, thus, increased the sensitivity of the method and also achieved baseline separation.

3.2.2 Precision and accuracy. In order to evaluate the precision of the developed method, a series of working mixture solutions containing $0.5 \mathrm{mg} \mathrm{L}^{-1}$ and $0.1 \mathrm{mg} \mathrm{L}^{-1}$ of TMT, TBT, DBT, and MBT, respectively, was tested at least for five times. The values of relative standard deviation (RSD) for retention times, peak areas and peak heights were shown in Table 1. The RSD ranged from $5.66 \%$ to $8.38 \%$ for peak areas $(n=5)$, which indicated a good reliability. The RSD values for migration time obtained in analysis of intra-day and inter-day precisions (Table 1). The RSD values were in the range of $2.1-3.1 \%$ (intra-day) and $2.4-3.4 \%$ (inter-day) respectively. The values of RSD were similar to those obtained by the CTAB coating method. ${ }^{27}$ The precision of the method was considered acceptable and helped the identification of the analytes in the CE speciation analysis. The semi-permanent coating is stable. In the present study, the capillary could be used about 1-day period without ever regenerating or refreshing the capillary, and the stability of DDAC coating was also proven to be excellent for aqueous CE separations. ${ }^{18,19}$ The reproducibility for capillary-to-capillary was also acceptable. The RSD values of migration times for capillary-tocapillary were in the range of $8.1 \%-10.7 \%(n=5)$. As a measurement of accuracy of the method, one certified reference material, CRM 477 (mussel tissue) was analyzed (Table 2) after sample preparation. Good agreement was achieved between the certified values (2.20 \pm 0.19 (TBT), $1.54 \pm 0.12$ (DBT), $1.50 \pm$ 0.28 (MBT)) and the determined value (1.81 \pm 0.13 (TBT), 1.36 \pm 0.16 (DBT), $1.17 \pm 0.18$ (MBT) $(n=3)$. The precision for the determination was $8.6,7.8$ and $18.7 \%$ for TBT, DBT and MBT, respectively.

\subsection{Sample analysis}

In order to evaluate the application of the semi-permanent coating CE-ICP-MS method, further experiments were conducted to determinate organotin compounds in real environmental samples. Water, grape wine and sea food (Mya arenaria Linnaeus) were chosen as different matrix samples. 
Table 2 Analysis results of organotin compounds in the certified reference material (CRM 477)

\begin{tabular}{lll}
\hline & $\begin{array}{l}\text { Concentration } \\
\text { found/mg kg }\end{array}$ & $\begin{array}{l}\text { Certified } \\
\text { value/mg kg }\end{array}$ \\
\hline TMT: $\operatorname{Sn}\left(\mathrm{CH}_{3}\right)_{3}{ }^{+}$ & - & - \\
TBT: $\mathrm{Sn}\left(\mathrm{C}_{4} \mathrm{H}_{9}\right)_{3}{ }^{+}$ & $1.81 \pm 0.13$ & $2.20 \pm 0.19$ \\
DBT: & $1.36 \pm 0.16$ & $1.54 \pm 0.12$ \\
$\mathrm{Sn}\left(\mathrm{C}_{4} \mathrm{H}_{9}\right)_{2}{ }^{2+}$ & $1.17 \pm 0.18$ & $1.50 \pm 0.28$ \\
MBT: $\operatorname{Sn}\left(\mathrm{C}_{4} \mathrm{H}_{9}\right)^{3+}$ & & \\
\hline
\end{tabular}

3.3.1 Determination of organotin compounds in water samples. A tap water sample (collected from the lab) and two river water samples (collected from two places of Liuyang River, China) were prepared and analyzed by the developed method as mentioned above. Organotin compounds were not detected in all of the water samples. Recovery tests were performed and two levels of concentration $\left(0.1 \mathrm{mg} \mathrm{L}^{-1}, 0.5 \mathrm{mg} \mathrm{L}^{-1}\right)$ of the standard mixture were spiked to all the water samples. Each of the samples were analyzed in triplicate. All the values of recovery were shown in Table S3. $\dagger$ The recoveries of the analytes ranged from $73.0 \%$ to $90.8 \%\left(0.1 \mathrm{mg} \mathrm{L}^{-1}\right.$ as $\left.\mathrm{Sn}\right)$, and $99.7 \%$ to $106 \%\left(0.5 \mathrm{mg} \mathrm{L}^{-1}\right.$ as $\left.\mathrm{Sn}\right)$. The $\mathrm{RSD}$ of the two river water samples were from $2.3 \%$ to $10.8 \%$. Fig. S4 (A) and S4 (B) are the electropherograms of the tap water and tap water spiked with $0.5 \mathrm{mg} \mathrm{L}^{-1}$ (as $\mathrm{Sn}$ ) samples, respectively. $\dagger$ It is obvious that the analytes contained in the river water samples were too low to be monitored. However, low LODs (0.037 to $0.112 \mathrm{mg} \mathrm{L}^{-1}$ ) of the optimized method ensure the positive quantification of the organotin compounds in contaminated water samples as previously published. ${ }^{28}$ Besides, the recoveries of the analytes in spiked analyte mixture and good signal to noise ratios $(\mathrm{S} / \mathrm{N}$, $0.5 \mathrm{mg} \mathrm{L}^{-1}$ as $\mathrm{Sn}$ for each) also showed the valid performance of the optimized separation parameters.

3.3.2 Determination of organotin compounds in wine samples. Other sample analysis, such as dry red wine sample analysis was also conducted by the developed method to quantify the organotin compounds contained. After the preparation, the samples were analyzed by the CE-ICP-MS system. MBT (0.325 $\mathrm{mg} \mathrm{L}^{-1}$ ) was monitored in the grape wine sample. Standard addition experiments of $0.5 \mathrm{mg} \mathrm{L}^{-1}$ (as $\mathrm{Sn}$ ) of each organotin compound to grape wine sample also have been performed. As illustrated in Table $\mathrm{S} 4, \dagger$ it can be seen that the recoveries of TMT and TBT were $98.7 \%$ and $96.8 \%$ while the recoveries of DBT and MBT were $62.3 \%$ and $31.8 \%$, respectively. Low recoveries of DBT and MBT may be attributed to the interference of sample matrix during separation of $\mathrm{CE}$ and analyte loss in sample preparation.

3.3.3 Determination of organotin compounds in mussel tissue samples. Mya arenaria Linnaeus is a bivalve halobios and was considered as one kind of bioindicator for the TBT accumulation in bivalves. In order to validate the CE-ICP-MS method to separate organotin compounds contained in complex biological samples, Mya arenaria Linnaeus samples and the standard mussel tissue reference material (CRM 477) were examined after microwave-assistant extraction as described in Experimental section.

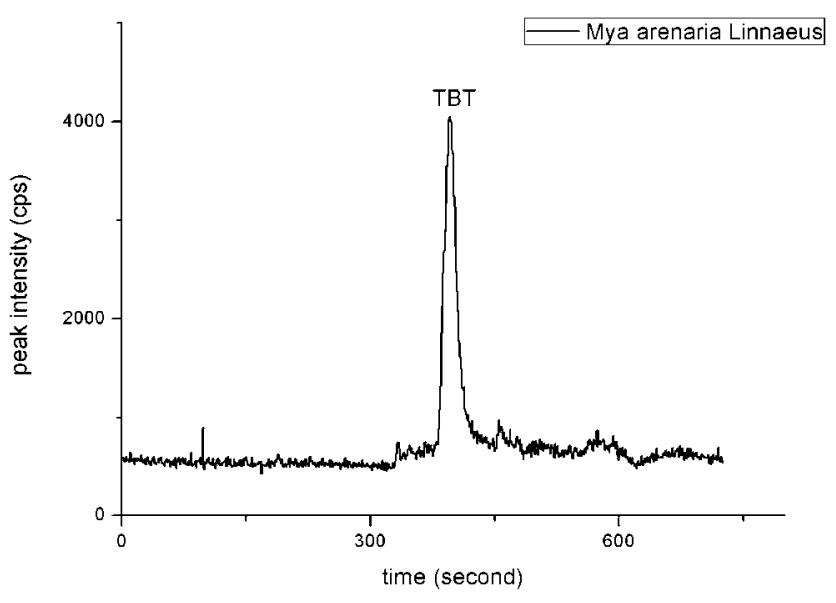

Fig. 4 Electropherogram of Mya arenaria Linnaeus samples. The data was obtained by the developed CE-ICP-MS method under optimized condition.

Three parallel Mya arenaria Linnaeus samples were tested, each was analyzed in triplicate. Fig. 4 is a typical chromatogram for organotins in Mya arenaria Linnaeus samples. Only TBT could be detected in the samples. The concentration level (ug g ${ }^{-1}$ as $\mathrm{Sn}$ ) of the tin compounds contained in samples was $1.02 \mu \mathrm{g} \mathrm{g}^{-1}$. The RSD of the three parallel was $7.6 \%$. It is the first time that organotin compounds in Mya arenaria Linnaeus samples were detected using the semi-permanent coating of the CE-ICP-MS method. Standard addition experiments also have been performed (Table S4). $\uparrow$ Two levels of concentration $(0.1$, $0.5 \mathrm{mg} \mathrm{L}^{-1}$ ) of the standard mixture were spiked. The recoveries of the four organotin compounds ranged from $68.6 \%$ to $80.0 \%$ $\left(0.5 \mathrm{mg} \mathrm{L}^{-1}\right)$ and $61.7 \%$ to $81.7 \%\left(0.1 \mathrm{mg} \mathrm{L}^{-1}\right)$. Higher spike recoveries could be obtained by adding agents such as tropolone and diethyldithiocarbamate, which would increase extraction efficiency in the sample preparation procedures. But it might influence the CE separation efficiency and stability. Thus, improvement of preparation section is still needed for the application of the CE-ICP-MS method to other important environmental matrixes.

\section{Conclusions}

A DDAC coating-CE-ICP-MS method was developed for the separation of four organotin compounds, and the method was sensitive and reproducible. DDAC semi-permanent coating procedure was simple and it could effectively enhance separation efficiency and improve the LODs of organotin compounds by reducing the adsorption on the inner wall of capillaries. Key separation parameters such as ionic strength and electrolyte composition were optimized and low LODs $\left(0.037 \mathrm{mg} \mathrm{L}^{-1}\right.$ (TMT) $-0.112 \mathrm{mg} \mathrm{L}^{-1}$ (MBT)) was obtained. The optimized method was applied for the analysis of the four organotin contaminants in the standard mussel tissue reference material (CRM 477) and several environment samples. Good agreement was achieved by determined values and certified values and $0.325 \mathrm{mg} \mathrm{L}^{-1}$ MBT in red wine and $1.02 \mu \mathrm{g} \mathrm{g}^{-1}$ (as Sn) TBT in Mya arenaria Linnaeus were firstly detected using the DDAC coating CE-ICP-MS system. 


\section{Acknowledgements}

This work was jointly supported by the National Basic Research Program of China (2009CB421605), and National Natural Science Foundation of P. R. China (20677069, 20921063 and 20937002).

\section{References}

1 C. J. Evans and S. Karpel, Organotin compounds in modern technology, Elsevier; Distributors for the U.S. and Canada, Elsevier Science Pub. Co., Amsterdam; New York, New York, N.Y, 1985.

2 G. B. Jiang, J. Y. Liu and Q. F. Zhou, Environ. Sci. Technol., 2004, 38, 4349-4352.

3 P. J. Craig, Organometallic compounds in the environment, J. Wiley, West Sussex, England; Hoboken, NJ, 2003.

4 F. E. Brinckman, J. M. Bellama and American Chemical Society, Division of Inorganic Chemistry, Organometals and organometalloids: occurrence and fate in the environment: based on symposium sponsored by the Division of Inorganic Chemistry at the 175th meeting of the American Chemical Society, Anaheim, California, March 13-17, 1978, American Chemical Society, Washington, 1978.

5 K. Fent, Crit. Rev. Toxicol., 1996, 26, 1-117.

6 M. D. Muller, Anal. Chem., 1987, 59, 617-623.

7 J. M. Liu, G. B. Jiang, J. Y. Liu, Q. F. Zhou and Z. W. Yao, Bull. Environ. Contam. Toxicol., 2003, 70, 219-225.

8 R. Ritsema, T. de Smaele, L. Moens, A. S. de Jong and O. F. X. Donard, Environ. Pollut., 1998, 99, 271-277.

9 J. Heroult, T. Zuliani, M. Bueno, L. Denaix and G. Lespes, Talanta, 2008, 75, 486-493.
10 M. G. Ikonomou, M. P. Fernandez, T. He and D. Cullon, $J$. Chromatogr., A, 2002, 975, 319-333.

11 G. Alvarez-Llamas, M. D. de laCampa and A. Sanz-Medel, $\operatorname{Tr} A C$, Trends Anal. Chem., 2005, 24, 28-36.

12 G. S. Zhai, J. F. Liu, L. Li, L. Cui, B. He, Q. F. Zhou and G. B. Jiang, Talanta, 2009, 77, 1273-1278.

13 K. S. Whang and C. W. Whang, Electrophoresis, 1997, 18, 241-246.

14 Y. T. Lee and C. W. Whang, J. Chromatogr., A, 1996, 746, 269-275.

15 L. Guo, F. M. Matysik and P. Glaser, Anal. Bioanal. Chem., 2004, 380, 669-676.

16 J. L. F. Feng Han and Phyllis R. Brown, J. Chromatogr., B: Biomed. Sci. Appl., 1995, 669, 103-112.

17 E. Pobozy, B. Glod, J. Kaniewska and M. Trojanowicz, J. Chromatogr., A, 1995, 718, 329-338.

18 W. L. Wei, B. Y. Guo and J. M. Lin, J. Chromatogr., A, 2009, 1216, 1484-1489.

19 M. M. Yassine and C. A. Lucy, Anal. Chem., 2005, 77, 620-625.

20 J. E. Melanson, N. E. Baryla and C. A. Lucy, Anal. Chem., 2000, 72, 4110-4114.

21 B. Michalke, J. Anal. At. Spectrom., 1999, 14, 1297-1302.

22 B. Michalke, Electrophoresis, 2005, 26, 1584-1597.

23 B. H. Li and X. P. Yan, Electrophoresis, 2007, 28, 1393-1398.

24 A. Standler, G. Koellensperger, W. Buchberger, G. Stingeder and S. Hann, Electrophoresis, 2007, 28, 3492-3499.

25 J. A. Day, S. S. Kannamkumarath, E. G. Yanes, M. Montes-Bayon and J. A. Caruso, J. Anal. At. Spectrom., 2002, 17, 27-31.

26 C. Pellegrino, P. Massanisso and R. Morabito, $\operatorname{Tr} A C$, Trends Anal. Chem., 2000, 19, 97-106.

27 L. P. Yu, Anal. Lett., 2007, 40, 1879-1892.

28 G. B. Jiang, J. Y. Liu and K. W. Yang, Anal. Chim. Acta, 2000, 421, $67-74$. 